
IP Periodica Polytechnica
Civil Engineering

61(3), pp. 398-411, 2017

https://doi.org/10.3311/PPci.9833

Creative Commons Attribution (i)

EDUCATIONAL PAPER

\section{Random Parameters and Sources of Uncertainty in Practical Fire Safety Assessment of Steel Building Structures}

\author{
Paweł A. Kró $1^{1 *}$
}

Received 30 July 2016; Revised 07 November 2016; Accepted 05 December 2016

\begin{abstract}
The purpose of this article is to identify sources of uncertainty and outline the basis of probabilistic safety assessment of steel structures exposed to fire conditions. Awareness of the existence of unavoidable uncertainties and non-deterministic nature of many phenomena and parameters may be essential for a proper understanding of the issues of structural reliability in the event of extreme or accidental actions.

The study highlights various sources of uncertainty associated with practical fire safety assessment of steel structures which significantly affects the reliability of estimates. Universal factors influencing random structural resistance in all design conditions were separately described. A significant part of the paper is devoted to issues that relate only to an accidental design situation of fire. Reference was made to the uncertainty of the analytical model describing the bearing capacity of the structure in terms of probabilistic approach. A synthetic summary on the impact of the method of carrying out material testing in elevated temperatures on the strength parameters, was made. In the conclusion it has been underlined that, inter alia, due to the lack of relevant statistical data, conducting a fully reliable probabilistic analysis of random structural resistance under fire is currently not possible. A unique part of the paper contains the proposed model of probability-based analytical approach to assess random structural resistance in fire conditions, incorporating an authorial modification to models used for standard conditions.
\end{abstract}

\section{Keywords}

fire, fire safety, structural element, steel structure, uncertainty, reliability, probability-based analytical approach, probability-based structural analysis, random resistance, random variable, deterministic value, high temperature analysis

\footnotetext{
${ }^{1}$ Division of Metal Structures, Institute of Building Engineering, Faculty of Civil Engineering, Warsaw University of Technology

PL-00637 Warsaw, Al. Armii Ludowej 16, Poland

"Corresponding author email: p.krol@il.pw.edu.pl
}

\section{Introduction}

In the classical, deterministic approach to the structural design, it is assumed that all the basic input data, such as dimensions of elements or strength properties of structural materials are the parameters of specific, unchanging values, quoted as nominal values in guides, tables and codes. Computational models used assume that the analysed structural elements will, in the actual structure, maintain the ideal geometry, shapes of sections, straightness of members, and ultimately the method of construction, assembly and operation will be fully compatible with the design assumptions. It is further anticipated, regardless of the technological processes and potential inaccuracy of construction and assembly, that the structure built will be free of any residual stresses and the quality of products used in the construction of the structure will be no worse than designed. This assumption is convenient but usually untrue. Each building structure has some imperfections, practically impossible to avoid, which can be classified as geometric, technological or structural imperfections. All of them are of random nature and a large part of them have already been well recognized in the statistical sense. Thus, they may be treated in a probabilistic approach to the design, as random variables described by a specific type of distribution, with a known degree of dispersion around the mean value.

One should be fully aware that all the imperfections in relation to the assumed ideal state exert a negative impact on the actual structural resistance, causing - in every case its decrease. In an accidental situation, they may show their impact in a less expected or sometimes even unexpected way, much earlier than it is the case in a normal design situation.

This article is intended to draw attention of the engineering industry to various types of uncertainty that may be encountered by a designer or a person responsible for the assessment of the technical condition of steel structures, especially those subjected to fire actions. Awareness of the existence of unavoidable sources of uncertainty may be crucial for proper performance of professional duties with the sense of absolute reliability and responsibility. 


\section{Random imperfections of structural members 2.1 Technological imperfections}

Technological imperfections are usually understood as internal residual stresses, unevenly distributed within the cross-section of the member and along its length. They are remnants of technological processes, to which the raw material is subjected in the steelworks during the process of rolling or a welded structural member in the production plant, before its actual assembly. In most cases, technological imperfections derive from thermal processes, the impact of which was not cancelled in the subsequent tempering or standardization processes. In the case of large differences in the values of residual stresses of opposite signs, they may even lead to longitudinal deformation of the elements or cross-sectional deformation of these members, which often happens in the case of improper selection of welding technology and sequence. The presence of residual stresses of high values may lead to a completely unexpected local damage to the structural member (e.g. within the heat affected zones in the vicinity of welds), in the case of summing up with the stresses caused by service loads in a situation where their total value exceeds the limit of the material resistance. Technological imperfections are dangerous in as much as that, although they do not cause visible deformation of members, they usually cannot be discovered before a failure with the "naked eye".

\subsection{Geometric imperfections}

Another type of imperfections occurring in steel structural members are the geometric imperfections. This group may include both imperfections within the cross section of a structural member and deformations along its length. The group of imperfections within the cross section may comprise those related to e.g. a non-conformity of dimensions (width and thickness) of section component walls with their nominal values and non-compliance of retaining shapes, manifested for instance by twisting of the walls (i.e. flanges and webs) or the lack of proper angles between them. It was observed that geometric imperfections of hot-rolled steel sections had been caused directly by the roll wear and the resulting clearance within the bearing points of rolls. Statistic surveys showed that I-beam flanges tend to thin out, whereas the webs - to thicken. As a result of thinning of the flanges, the cross section moment of inertia is noticeably reduced, which adversely affects its bending moment resistance. In the case of these random changes of geometrical features of the sections, they may be large enough to be taken into account in the resistance estimation. In the case of design of steel structures exposed to fire conditions, they can affect both the value of the critical temperature (due to the change in the real value of cross-section degree of resistance utilization factor $-\mu_{0}$ ) and lead to errors in estimates of temperature of steel members subjected to fire actions (due to the change in the section factor $-A_{m} / V$ ). Thus, they can lead to erroneous estimation of structural safety.
Among the geometric imperfections occurring along the length of a structural member, they can be classified for example into: the element bending in one or both planes, section twisting, local buckling and bending of shape of flanges, webs, and the like. The consequence of these imperfections is a change in the arrangement of internal forces in relation to the theoretical model adopted, whereas the presence of previously unforeseen structural eccentricities also leads to the formation of the second-order moments. The presence of these phenomena may lead to an early achievement of the limit state by the member, associated with the critical state or earlier than expected occurrence of excessive deformations, unacceptable from a utility point of view. A certain confirmation of this statement is provided in the example cited at the final part of this article, illustrating the effect of various types of imperfections on the quality of the results of numerical analysis performed for a column in compression, subjected to the influence of simulated fire temperature. Moreover, even local deformations, such as local buckling or bending of the section component walls - particularly in the case of members subject to compressive forces and exposed to lateral buckling - may cause the initiation of these adverse effects with the load potentially safe for a corresponding structure with perfect geometry. It may even be attempted to formulate a general statement that the resistance of the compressed structures with imperfections (not only geometric) is in any case smaller, than the resistance of a perfect structure.

In the design codes, the effect of certain limited imperfections was taken into account in the form of properly calibrated instability coefficients. Depending on the degree of sensitivity of an element to the initial random geometric and technological imperfections of compression members, the code EN 1993-1-1 [1] proposes four buckling curves. They were determined by analysing the model of the compression member with the initial equivalent curves $w_{0}$, which are, respectively, for the buckling curve "a" $-w_{0}=l / 500$, for the curve " $\mathrm{b} "-w_{0}=l / 250$, , c" $" w_{0}$ $=l / 200$ and " $\mathrm{d}$ " $-w_{0}=l / 150$. In addition to the imperfections arising in the milling and rolling process, among this group of structural imperfections may also be classified geometrical deviations resulting from the quality of fabrication process of the structural elements in the manufacturing plant (e.g. accuracy of marking holes for screws) and the conditions of its assembly. Thus, in the case of a structure with a greater complexity it is advised to carry out its pre-assembly in the manufacturing plant, which is intended to eliminate any alterations or "ffitting" and "stretching" the structure on the site. Such activities are unacceptable because they generate internal forces with values difficult to earlier predict and considerably change the stability characteristics of the structure.

Standards regarding the basic conditions for the construction and acceptance of structures indicate permissible limits of initial geometrical, technological, construction and assembly defects, which can be regarded as not significantly reducing 
the structural resistance and level of safety. Such reasoning, however, is characteristic to the deterministic approach for the structural design, whereas in the probabilistic approach, any geometric imperfections should be taken into account by treating them as random variables with specified distribution and degree of variation.

\subsection{Material and structural imperfections of steel products and their impact on key strength parameters}

In the elastic-plastic material model, widely adopted in the design of steel structures, two parameters seem to be the most important: the yield strength of steel $-f_{y}$ and Young modulus $E$. The first value has a significant impact on the estimation of the member load-carrying resistance within the meaning of the Ultimate Limit State (ULS), whereas the second one - on the assessment of their deformation under load, within the meaning of the procedures adopted in relation to the Serviceability Limit State (SLS). Both these values are random in the probabilistic approach and their distributions and statistics at ambient temperature - due to a relatively high number of recorded tests - are fairly well known and understood. Anyone who has ever performed a static tensile test of steel samples is fully aware of the fact that in practice, in principle, it is not possible to achieve identical values of the ultimate force or the values of the elastic modulus for a series of separate samples, and the range of the measured values is, in many cases, significant. The random nature of the values is directly affected by the existing structural imperfections, manifesting themselves by uneven distribution of mechanical properties of the material - both within the cross-section and along the length of the steel product. The range of results of properly designed and conducted testing (i.e. static tensile testing of metals) is due to the differences in chemical composition, contained impurities and inclusions, uneven distribution of unwanted components resulting in part from the steel casting technology and in part - from the method of its processing in the steelworks. Hot rolling, for example, causes deposition of the impurities in the centre of the volume of the finished product, due to slower cooling of these areas. In the case of rolled sections, these are usually the contact areas of flanges and web, resulting inter alia in a recommendation for non-performance of welds in areas where the material is assumed to be of poorer quality. In these areas, residual stresses are also often concentrated, being remnants of the rolling process. The rolling process also leads to natural deformation of crystals, which causes certain anisotropy of mechanical properties of a seemingly isotropic construction material. For this reason, it is recommended to shape the structure so that the direction of the dominant strain coincide with the rolling direction, where the mechanical properties remain the best. Thickness of the members is also not without significance for the mechanical properties. Thicker members, made of the same material, are weaker than thinner members. These differences in strength can be quite important, therefore they are reflected in the design standards in the form of various nominal yield strength values given for different thickness groups of products.

Continuing the subject of the random nature of the selected strength parameters, information on the nominal value of the modulus of elasticity adopted in the design standards can be given here as an interesting fact, which some standards specify at $E=205 \mathrm{GPa}$, while others - at a bit higher $E=210 \mathrm{GPa}$. As stated in [2], accurate static tests made and collected over several years have shown that the average value of the modulus of elasticity is $\bar{E}=\mu_{E}=204 G P a$, with the coefficient of variation of $V_{E}=\sigma_{E} / \mu_{E}=0.013$, where $\sigma_{E}$ is a standard deviation about the mean value. This allows the determination of the characteristic (nominal) value of the elastic modulus of steel (as $95 \%$ quantile of the lognormal distribution with the tolerance rate of $t=1.64)$ :

$E_{k}=E_{\text {nom }}=\bar{E}-t \cdot \sigma_{E}=\mu_{E}-t \cdot \sigma_{E}=\mu_{E}-t \cdot V_{E} \cdot \mu_{E}=$ $=\mu_{E}\left(1-t \cdot V_{E}\right)=204 \cdot(1-1.64 \cdot 0.013)=200 \mathrm{GPa}$

The difference at the expense of safety between the actual value resulting from the statistics and the one that was adopted in the design codes, e.g. in EN 1993-1-1 [1] equals (210 - 200) / $200 \cdot 100 \%=5 \%$. It was however considered by scientists as a negligible difference.

A completely separate issue is the question of variability in material strength parameters: yield strength $-f_{y}$ and Young modulus $-E$, during the exposure to fire temperatures, to which the next section is entirely devoted.

It should also be noted that the probabilistic approach most frequently uses the lognormal distribution for the description of the random nature of the material properties of steel and in the case of small populations - normal distribution. This information is important from the point of view of the material resistance reliability model description applied, which will be expanded on later in the paper.

\section{Material properties of structural steel at increased temperature}

The most important information on the mechanical and thermal properties of structural steel and their variability depending on the temperature is provided in the code EN 1993-1-2 [3].

When checking resistance of the steel structures at the normal temperature (also referred to interchangeably as the ambient temperature), it is assumed that the yield strength $-f_{y}$ and Young modulus $-E$ have both constant values. In structural safety assessment at fire temperatures, degradation of these parameters with increasing temperature of the member is taken into account, adopting for calculations of a current value, appropriate to the steel temperature determined according to the code procedures. The values of actual (effective) yield strength, proportional limit and Young modulus 
at increased temperatures are given in the code indirectly, by means of the tabulated values of the reduction factors $k_{y, \theta}$, $k_{p, \theta}$ and $k_{E, \theta}$, where $k_{y, \theta}=f_{y, \theta} / f_{y}$ means, respectively, the ratio of the effective yield strength to the yield strength at the temperature of $20^{\circ} \mathrm{C}, k_{p, \theta}=f_{p, \theta} / f_{y}$ - the ratio of the proportional limit to the yield strength at the temperature of $20^{\circ} \mathrm{C}$, whereas $k_{E, \theta}=E_{a, \theta} / E_{a}$ - the ratio of the Young modulus at a given temperature to the modulus of elasticity at the temperature of $20^{\circ} \mathrm{C}$.

Apart from the summarizing table and its accompanying graph, the code does not provide any analytical formula allowing for precise calculation of the current value of the reduction factors for a specific temperature, recommending the use of linear interpolation for intermediate temperature levels, not listed in the table. The degradation method adopted in the Eurocode is a polygonal chain for each of the factors, consisting of several line segments. A very similar way to describe the variability function of reduction factors was adopted in the American documents ANSI/AISC 360-10 [4] and AISC Steel Design Guide 19 [5]. Some slight differences in the course of the variability functions stem rather from the conversion of imperial units used in the United States into SI units, than from any other reason.

It is worth noting that there are some developed estimations of factors reducing values of yield strength and modulus of elasticity relating not to polylines but to continuous lines adapted to them, as suggested by the standards [3], [4] and [5], however, they have never been formalized in the form of documents widely recognized and accepted by the research community.

For a change, an analytical method for determining variability of these factors is provided in such standards as the New Zealand standard NZS 3404: Part 1:1997 [6], the previous Polish standard PN-B-03200:1990 [7] and the document of the Technical Committee of the European Convention for Constructional Steelwork ECCS [8].

Equations specified in the New Zealand standard [6] are based on findings of the French research institute CTICM. They are to some extent, in a quantitative sense, similar to the recommendations that can be found in the aforementioned previous version of the national Polish standard [7] or findings developed by the ECCS-TC3 [8].

Alternative formulas, but referring to the types of steel grades used in the manufacture of thin-walled structural members, were presented by Lee, Mahendran and Mäkeläinen in their paper [9].

It should be noted that not only the differences in a purely quantitative dimension, but also some qualitative differences are noticeable in this respect between the European standard [3] as well as the previous Polish standard [7]. In the case of the standard [3], yield stress value $f_{y, \theta}$ decreases along with the temperature rise more slowly than in the case of the modulus of elasticity $E_{a, \theta}$, whereas in the case of the standard [7], the situation is opposite. To compare differences in the suggested description methods values of reduction factors determined according to methods suggested above were illustrated in Fig. 1 and Fig. 2.

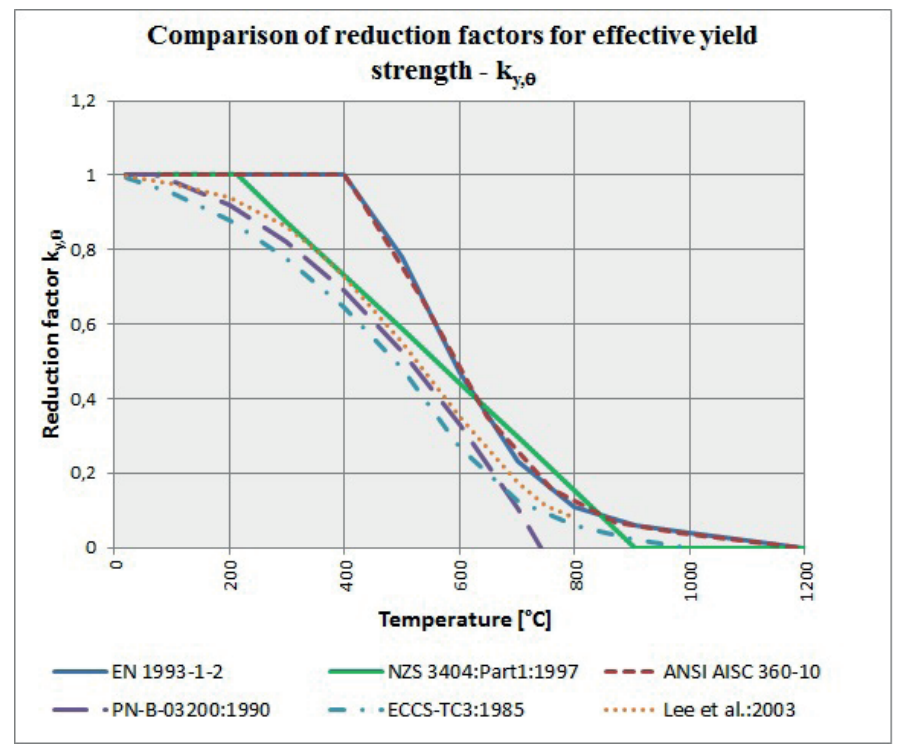

Fig. 1 Reduction factors for effective yield strength (relative to $f_{y}$ ) - comparison

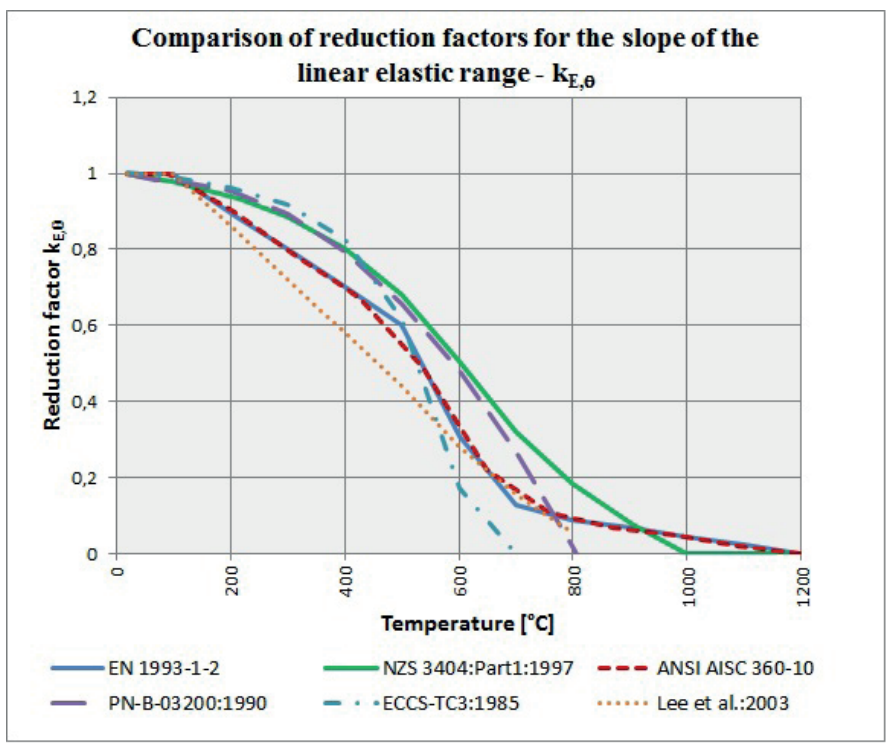

Fig. 2 Reduction factors for the slope of the linear elastic range (relative to $E_{y}$ ) - comparison

Attention should be drawn to huge differences in the values of reduction factors determined on the basis of standardization documents cited above and consequential differences in the final design values of the member resistance or estimated deflection. For example, the yield strength $f_{y, \theta}=f_{y, 400^{\circ} \mathrm{C}}$ at $400^{\circ} \mathrm{C}$, according to the European code [3] and the American standards [4] and [5] is equal to the initial value $f_{y}$, as determined for normal conditions, whereas when it is determined according to ECCS document [8], it is only $65 \%$ of the base value.

Similarly, when looking at the discrepancies in the assessment of the Young modulus values at $600^{\circ} \mathrm{C}$, according to the European code [3], the value $E_{a, \theta}=E_{a, 600^{\circ} \mathrm{C}}$ equals $31 \%$ of the initial value $E_{a}$, as determined for normal conditions, according to the ECCS document [8] respectively only $17.3 \%$ of the initial value, whereas according to the New Zealand standard [6] as much as $50.5 \%$ of the base value. 
Is not entirely clear where exactly such differences stem from, ranging from several to dozens of percent, but when referring it to the $5 \%$ difference between the actual value of Young's modulus resulting from the statistics and that adopted in the design standards as the nominal value, mentioned above as an interesting fact, it can be considered as negligible or even irrelevant.

Such a significant difference in the values of the corresponding reduction factors may be due to several issues. It should be noted that the New Zealand standard is a document older than the Eurocode, thereby the research methodology used in determining the value of yield strength and Young's modulus at increased temperatures could be different than the one currently used. Also the quality of steel as the material itself could be then lower, than compared to the contemporary grades of steel. The method of testing and the steel heating rate have a significant impact on the actual values of steel strength and material parameters at increased temperatures, established by experiments. This topic will be further developed in the later part of the work, but it is useful now, when comparing the differences in the assessment of safety-critical structural strength parameters, to realize the relativistic non-deterministic nature of the majority of parameters that ultimately exert a significant impact on the level of uncertainty associated with the assessment of safety and reliability of engineering structures using currently available assessment procedures.

\section{Uncertainty of the model describing structural resistance}

The simplest meaning of the structural resistance is the structure capacity to carry loads. It usually depends on the load-carrying resistance of its individual components and connections (or in broader meaning - joints) between them. Load-carrying capacity (resistance) of the member, usually marked in the literature and standards of design with the letter $R$ (in general terms) or as a value corresponding to a particular type of internal forces with the subscript $R$ (e.g. $M_{R}, N_{R}, V_{R}$, or alternatively $M_{R d}, N_{R d}, V_{R d}$-in relation to the design values), is in the classic design understood as a direct function of the material strength (defined, depending on the standard, as the yield strength - $f_{y}$, design resistance $-f_{d}$, etc.), the relevant geometrical parameters of the section (cross-sectional area - for axial and transverse forces, section modulus - for bending elements, etc.) and longitudinal dimensions (length, span), which are the measure of the member slenderness, responsible for the risk of loss of global stability. In the case of members with slender walls, a separate measure of slenderness is responsible for the risk of loss of local stability. In the classical approach to the design, using procedures specified by standards, all of the aforementioned values are taken as deterministic ones of fixed, predetermined value. In fact - most of them can and even should be treated as separate random variables, since each of them are accompanied by some variability, confirmed by the results of material tests conducted in the laboratory, dimensional deviations measured (within acceptable tolerances) or imperfections resulting from the manufacturing accuracy. Variability of these parameters in the design is taken into account in the form of the probabilistic approach.

Sources of uncertainty affecting the final value of the member resistance, as set out in a probabilistic manner, can be seen in each of the three areas concerning directly: material properties (structural imperfections), quality of fabrication (geometric and technological imperfections) and the method of structural analysis or degree of simplification of the calculation model (e.g. with respect to the static scheme adopted, the nature of the final elements used when conducting numerical analyses, etc.) in relation to the conditions prevailing in the real structural system. The issue of material and geometric imperfections was already sufficiently developed in the previous section and may be referred to for review. Rather, the attention should be focused on the imperfections of the analytical model.

Inaccuracies related to the method of analysis are the result of the simplifications adopted during the calculation of the structure in terms of computational methods chosen (more or less precise), simplification of the actual static schemes, simplifying assumptions of a different nature, noticeably shortening analysis time, and finally - referring especially to the structural design in fire conditions - selection of the fire scenario, method of describing the function of fire gases temperature increase in time, method of analysis adopted, and, in consequence - reliability of thermal conditions to which the structure will be or has been exposed.

An excellent example of the ambiguity of fire thermal conditions assessment is shown in Fig. 3, presenting the temperaturetime dependence for the same room (fire compartment) in a residential building, based on the standard model and the parametric model of fire description. Depending on the choice of the function describing the fire gases temperature increase in time, quite inconsistent and highly divergent data for further analysis may be obtained, which may lead to errors already at the stage of initial assumptions, before even starting the actual process of structural safety assessment in given environmental conditions. Often the choice of a particular fire scenario itself (e.g. parametric fire) and the method of its description create additional sources of uncertainty in the assessment process of thermal conditions due to the fact that the course of function (including the heating rate of construction material) is dependent on a number of other parameters describing the environment of fire, most of which can be treated as separate independent random variables. Not all of them have been as yet sufficiently well analysed and described in the statistical sense. The most dynamic period of research devoted to fire safety of buildings falls in fact for the last dozen years, or perhaps the last decades, which means that we still know relatively little about the nature of the phenomena occurring during fire or this knowledge is incomplete. 


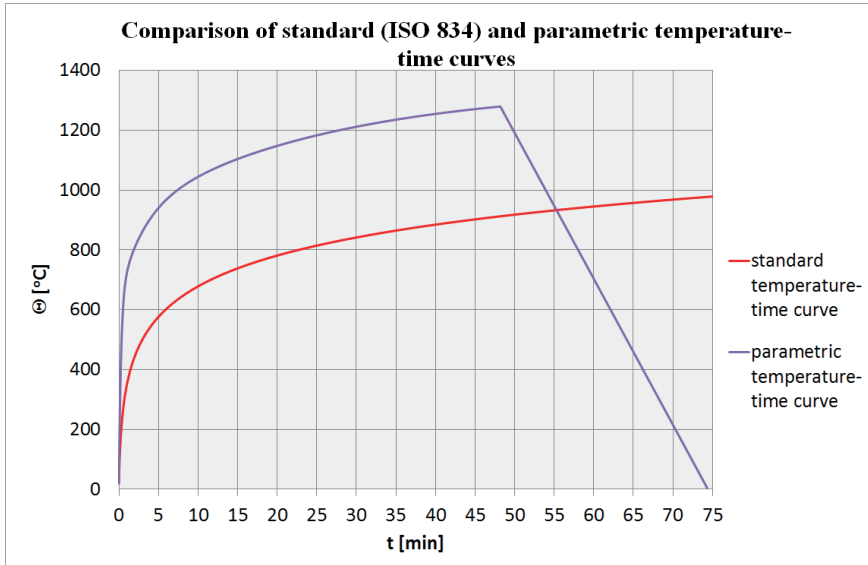

Fig. 3 Comparison of standard temperature-time and parametric temperaturetime curves

Variability of some parameters affecting directly or indirectly structural resistance was to some extent recognized and quantified by tests carried out in specialized laboratories, observation of existing facilities, measurements and experts' evaluations. Part of this information can be found in the available literature, but it is usually incomplete, some is still a subject of research, which is expensive and often difficult to be unambiguously interpreted or impossible to generalize because it refers to a specific configuration of the fire environment. The richest source of information on fire statistics, useful from the point of view of structural and construction design, can be found in already slightly archival American or Antipodean documents, e.g. [10], [11] or [12]. Most of the data contained therein relate to office buildings and residential buildings, however the usefulness of these data when attempting to transfer them directly to European conditions is not always possible. When it comes to European statistics, they are currently very poor and not particularly useful from a scientific point of view, and in some countries - due to the structure of emergency systems and services - they (i.e. statistical data related to fire events) are not collected at all.

\section{Probabilistic approach to the structural safety assessment}

The available literature provides many elements of the theory concerning the methods of structural analysis using probability theory achievements, including issues of uncertainty and reliability problems. It is impossible to mention all important papers that have noticeably influenced the development of this scientific discipline within this article, but among the world classics can be found, inter alia, papers by Hasofer and Lind [13], Rackwitz and Fiessler [14], Thoft-Christensen and Baker [15], Thoft-Christensen and Murotsu [16], Nowak and Collins [17], and Melchers [18], just to mention the most famous and popular ones. All these papers generally relate to the issues of reliability and probabilistic design of structures in the so called normal design conditions. They do not refer directly to the issue of structural reliability in case of fire, although some references to other accidental situations can be found there.
Some aspects of the probabilistic approach in fire design of steel structures were more recently touched on by Maślak [19]. He pointed to the need to use probabilistic methods in designing process, however, the information contained therein, does not fully fill in the niche in this area, both in the reference books and in awareness of the engineering industry.

The way of describing the resistance model uncertainty adopted in the American or Australian literature, e.g. [17], [18] is a bit different from the approach presented in the European literature, e.g. [2].

In the reliability approach to structural analysis, propagated in English literature, a popular way of modelling the resistance $R$ (e.g. $M_{R}, N_{R}, V_{R}$ ) is to treat it as a product of the nominal resistance $R_{n}$, understood hereinafter as a deterministic value used in a classic design and three additional parameters, taking into account the individual, previously mentioned sources of uncertainty that should be treated as random variables with a known distribution.

In mathematical terms, this is presented as follows:

$$
R_{\theta}(\varpi)=R_{n, \theta} M(\varpi) P(\varpi) E(\varpi) T(\varpi)
$$

where:

$R_{n}$ - is the nominal value of proper resistance understood as a deterministic value,

$M(\omega)$ - a "material factor" - is a parameter describing the method of variation of strength parameters of the structural material (material parameters), reflecting the structural imperfections. It is a factor defined as the ratio of actual to nominal material parameters (such as the ratio of yield strength, as determined by a static tensile testing of metals, to the value provided for in the design standards).

$P(\omega)$ - a "production factor" (also known as a "fabrication factor") - is a parameter describing the variability of parameters dependent on manufacturing and construction processes (e.g. dimensional imperfections), reflecting technological and assembly imperfections. It is a value defined as the ratio of the member's actual dimensions (determined on the basis of the member's direct measurements) to the nominal values of proper parameters (given in the tables or standards such as, for example, cross-sectional area, moment of inertia, the elastic or plastic modulus, and the like).

$E(\omega)$ - an "expert factor" (also known as a "professional factor") - is a parameter, taking into account the imperfections due to simplification within the accepted methods of structural analysis. The "expert factor" is defined as the ratio of the actual resistance of the member (specified e.g. on the basis of tests or observation of behaviour in real structures, measurements during the test loads etc.) to the nominal resistance, i.e. adopted on the basis of the results of the analysis conducted using a determined computational model. 
In the case of structural fire analysis, the resistance model description presented in the form of the Eq. (1) should be extended at least with the parameter $T(\omega)$ to form:

$$
R_{\theta}(\varpi)=R_{n, \theta} M(\varpi) P(\varpi) E(\varpi) T(\varpi)
$$

where:

$T(\omega)$ - a "thermal factor" - should be understood as the ratio of the actual temperature reached by the structural element during a real fire to the temperature value estimated on the basis of the fire scenario (model). It should be noted that in the case of steel members protected with fireproof insulation, variability of the thermal coefficient $T(\omega)$ may also be dependent on the variability of the insulation properties and must be estimated independently for each type of insulating material.

Due to the introduction of the thermal parameter (while assuming that the adopted method of description of the values of all the four parameters $M(\omega), P(\omega), E(\omega)$ and $T(\omega)$ are insensitive to changing thermal conditions) the actual structural resistance, like the value of the nominal resistance values automatically become temperature-dependent, due to a strong dependence of the current value of the yield strength - $f_{y, \theta}$ and Young's modulus $-E_{a, \theta}$, on the actual temperature conditions of the environment. However, from the viewpoint of the uncertainty of the assessment of the actual value of both these parameters, (compare Fig. 1 and Fig. 2), in fact the value $R_{n, \theta}$ in Eq. (2), is not to be regarded as the deterministic value but also as a random variable $R_{n, \theta}(\varpi)$, which leads to a change in the Eq. (2) to the following form:

$$
R_{\theta}(\varpi)=R_{n, \theta}(\varpi) M(\varpi) P(\varpi) E(\varpi) T(\varpi)
$$

In order to restore a similar logical structure to the relationship (3), characteristic for the Eq. (1), one more factor should be introduced - $D(\omega)$, understood as the "accuracy factor" of effective material parameters assessment $\left(f_{y, \theta}, E_{a, \theta}\right)$ for specified thermal conditions. Following the introduction of the accuracy factor $-D(\omega)$, the Eq. (4) takes the form of:

$$
R_{\theta}(\varpi)=R_{n, \theta} M(\varpi) P(\varpi) E(\varpi) T(\varpi) D(\varpi)
$$

where $R_{n, \theta}$ becomes a deterministic value, specifying the nominal resistance in specified thermal conditions. In the classical design approach, the nominal value of resistance in a fire situation $R_{n, \theta}$ is therefore described by the mathematical relationship between the other values understood here as deterministic values, namely strictly defined values, which in terms of resistance can be understood as for instance: $M_{f, \theta, R d}=W_{p l, y} \cdot f_{y, \theta} / \gamma_{M, f i}$ in the case of bending moment resistance of members protected against lateral-torsional buckling or $N_{f i, \theta, R d, t}=A \cdot f_{y, \theta} / \gamma_{M, f i}$ in the case of tensile elements (convention adopted in Eurocodes used), etc.

The relationship described by the Eq. (4) is a non-linear function, being the product of several independent random variables. By linearization of the function (4) by a Taylor series expansion and application of a simplification acceptable from a technical point of view, allowing the use in subsequent analyses of only first-order components, and assuming also that the random resistance of the member in an accidental design situation of fire is a random variable with lognormal distribution, calculated as the product of several random variables with lognormal distributions, using the Central Limit Theorem, relationships can be derived for:

- mean value $\mu_{R_{\theta}}$ of the random variable $R_{\theta}(\varpi)$ in the form of:

$$
\mu_{R_{\theta}}=R_{n, \theta} \mu_{M} \mu_{P} \mu_{E} \mu_{T} \mu_{D}
$$

where: $\mu_{M}, \mu_{P}, \mu_{E}, \mu_{T}, \mu_{D}$ are the mean values of the relevant parameters $M(\omega), P(\omega), E(\omega), T(\omega)$ and $D(\omega)$;

- bias factor $\lambda_{R_{\theta}}$ of the random variable $R_{\theta}(\varpi)$, defined as the ratio of the mean value of a given variable to its nominal value $\lambda=\frac{\mu}{\text { nom }}$ in the form of:

$$
\lambda_{R_{\theta}}=\lambda_{M} \lambda_{P} \lambda_{E} \lambda_{T} \lambda_{D}
$$

where: $\lambda_{M}, \lambda_{P}, \lambda_{E}, \lambda_{T}, \lambda_{D}$ represent bias factors of the following parameters: $M(\omega), P(\omega), E(\omega), T(\omega)$ and $D(\omega)$;

- coefficient of variation $V_{R_{\theta}}$ of the random variable $R_{\theta}(\varpi)$ in the form of:

$$
V_{R_{\theta}}=\sqrt{\left(V_{M}\right)^{2}+\left(V_{P}\right)^{2}+\left(V_{E}\right)^{2}+\left(V_{T}\right)^{2}+\left(V_{D}\right)^{2}}
$$

where: $V_{M}, V_{P}, V_{E}, V_{T}, V_{D}$ represent coefficients of variation of the relevant parameters $M(\omega), P(\omega), E(\omega), \mathrm{T}(\omega)$ and $D(\omega)$.

Statistics describing variability of parameters $M(\omega), P(\omega)$, $E(\omega)$, (i.e. their mean values, bias factors and coefficients of variation), based on the results of representative statistical surveys, are available in literature. One of the most credible list of these values could be found, for example, in [20], [21], and [22].

There is also possible a slightly different approach to the issue of uncertainties of the structural resistance model and the mathematical description of the problem. The available European literature is dominated by the concept of random resistance of a member, which in the general case (subject only to normal operating conditions of the structure) can be written down as follows:

$$
N(\varpi)=a(\varpi) C(\varpi) f(\varpi)
$$

where:

$a(\varpi)$ - means the ratio of the structural strain/stability model, understood as the factor of lateral torsional buckling, lateral buckling or local instability in the case of slender component walls of cross-sections (as the case may be);

$C(\varpi)$ - means a random geometric characteristics of the member cross section (e.g. cross-sectional area - $A(\varpi)$, section modulus - $W(\varpi)$, shear cross-sectional area $-A(\varpi)$, depending on the computational case and the analysed condition of the structure);

$f(\varpi)$ - random material strength, defined, respectively, as the yield strength $-f_{y}$ or the design strength $-f_{d}$, depending on the standards concerned. 
This way of describing resistance model in a probabilistic approach is suggested in the papers by, among others, Biegus [2] or Woliński and Wróbel [23]. The proposed description method takes into account all possible types of imperfections listed and described in the earlier sections of the paper, namely random strength parameters of material, random geometric characteristics of the member, random geometrical imperfections of the member axis, and random eccentrics of loads. By the applied way of reasoning, the member resistance is no longer a deterministic value of a strictly fixed magnitude but it becomes a probabilistic value as a function representing a combination of several random variables described by respective distributions.

For estimating the resistance while taking into account also accidental fire actions, the Eq. (8) becomes inaccurate, since it does not take into account the differences both in the description of the instability curves used in two different design situations (through the random ratio of the structural strain (stability) model) and random variability of material strength at increased temperatures. Using a sort of analogy, the Eq. (8) for an accidental design situation of fire could be expressed in the following form:

$$
N_{\theta}(\varpi)=a_{\theta}(\varpi) C(\varpi) f_{\theta}(\varpi)
$$

where:

$a(\varpi)$ - means the ratio of the structural strain/stability model at fire temperature;

$f_{\theta}(\varpi)$ - random material strength in a situation corresponding to the temperature conditions during a fire.

Using the philosophy adopted in the set of Eurocodes, random material strength at increased temperatures $-f_{\theta}(\varpi)$ can be expressed as the product $f_{\theta}(\varpi)=k_{\theta}(\varpi) f(\varpi)$, where $k_{\theta}(\varpi)$ means the random reduction factor of random material strength, thus the Eq. (9) takes the form of:

$$
N_{\theta}(\varpi)=a_{\theta}(\varpi) C(\varpi) k_{\theta}(\varpi) f(\varpi)
$$

or by referring it to the random resistance of the member in a normal design situation, as described by the Eq. (8):

$$
N_{\theta}(\varpi)=\frac{a_{\theta}(\varpi) k_{\theta}(\varpi)}{a(\varpi)} N(\varpi)
$$

To estimate the random resistance of a structural member, simplified methods (often called Level II probabilistic methods) are sufficient, which use only the knowledge of the two moments: (i) mean value of a given random variable $-\mu_{x}$ (as the first-order ordinary moment) and (ii) its variance $-\sigma_{x}^{2}$ (as the second-order central moment). In practice, also some other related values are used, such as: standard deviation $-\sigma_{x}=\sqrt{\sigma_{x}^{2}}$ and the coefficient of variation $-V_{x}=\frac{\sigma_{x}}{\mu_{x}}$.
If assumed, as previously, that the final product in the form of random resistance of the member in an accidental design situation of fire is a random variable with a lognormal distribution, calculated as the product (or quotient) of several random variables with lognormal distributions, then by analogy - for the variables described by the Eqs. (10) (or (11)), the relationship respectively describing the mean value $\mu_{N_{\theta}}$ of a random variable $N_{\theta}(\varpi)$, bias factor $\lambda_{N_{\theta}}$ or coefficient of variation $V_{N_{\theta}}$, can be written similarly to those previously shown in the form of equations (5), (6) and (7), and therefore:

- mean value $\mu_{N_{\theta}}$ of the random variable $N_{\theta}(\varpi)$ takes the following form:

$$
\mu_{N_{\theta}}=\mu_{a_{\theta}} \mu_{C} \mu_{k_{\theta}} \mu_{f}
$$

where: $\mu_{a_{\theta}}, \mu_{C}, \mu_{\theta}, \mu_{f}$, represent corresponding mean values of random variables $a_{\theta}(\varpi), C(\varpi), k_{\theta}(\varpi)$ and $f(\varpi)$;

- bias factor $\lambda_{N_{\theta}}$ of the random variable $N_{\theta}(\varpi)$ takes the following form:

$$
\lambda_{N_{\theta}}=\lambda_{a_{\theta}} \lambda_{C} \lambda_{k_{\theta}} \lambda_{f}
$$

where: $\lambda_{a_{\theta}}, \lambda_{C}, \lambda_{k_{\theta}}, \lambda_{f}$ represent bias factors of relevant random variables $a_{\theta}(\varpi), C(\varpi), k_{\theta}(\varpi)$ and $f(\varpi)$;

- coefficient of variation $V_{N_{\theta}}$ of the random variable $N_{\theta}(\varpi)$ takes the following form:

$$
V_{N_{\theta}}=\sqrt{\left(V_{a_{\theta}}\right)^{2}+\left(V_{C}\right)^{2}+\left(V_{k_{\theta}}\right)^{2}+\left(V_{f}\right)^{2}}
$$

where: $V_{a_{\theta}}, V_{C}, V_{k_{\theta}}, V_{f}$ represent corresponding coefficients of variation of random variables $a_{\theta}(\varpi), C(\varpi), k_{\theta}(\varpi)$ and $f(\varpi)$.

In addition, if the random variable $N_{\theta}(\varpi)$ has a lognormal distribution, then the random variable $\ln N_{\theta}(\varpi)$ has a normal distribution and in such case, the following relationships also occur:

$$
\begin{gathered}
\mu_{\ln N_{\theta}}=\mu_{\ln a_{\theta}}+\mu_{\ln C}+\mu_{\ln k_{\theta}}+\mu_{f} \\
\sigma_{\ln a_{\theta}}^{2}=\sigma_{\ln a_{\theta}}^{2}+\sigma_{\ln C}^{2}+\sigma_{\ln k_{\theta}}^{2}+\sigma_{\ln f}^{2}
\end{gathered}
$$

where:

$$
\begin{gathered}
\sigma_{\ln a_{\theta}}^{2}=\ln \left(1+\frac{\sigma_{a_{\theta}}^{2}}{\mu_{a_{\theta}}^{2}}\right)=\ln \left(1+V_{a_{\theta}}^{2}\right) \\
\sigma_{\ln C}^{2}=\ln \left(1+\frac{\sigma_{C}^{2}}{\mu_{C}^{2}}\right)=\ln \left(1+V_{C}^{2}\right) \\
\sigma_{\ln k_{\theta}}^{2}=\ln \left(1+\frac{\sigma_{k_{\theta}}^{2}}{\mu_{k_{\theta}}^{2}}\right)=\ln \left(1+V_{k_{\theta}}^{2}\right) \\
\sigma_{\ln f}^{2}=\ln \left(1+\frac{\sigma_{f}^{2}}{\mu_{f}^{2}}\right)=\ln \left(1+V_{f}^{2}\right) \\
\mu_{\ln a_{\theta}}=\ln \left(\mu_{a_{\theta}}\right)-\frac{1}{2} \sigma_{\ln a_{\theta}}^{2} \\
\mu_{\ln C}=\ln \left(\mu_{C}\right)-\frac{1}{2} \sigma_{\ln C}^{2}
\end{gathered}
$$




$$
\begin{gathered}
\mu_{\ln k_{\theta}}=\ln \left(\mu_{k_{\theta}}\right)-\frac{1}{2} \sigma_{\ln k_{\theta}}^{2} \\
\mu_{\ln f}=\ln \left(\mu_{f}\right)-\frac{1}{2} \sigma_{\ln f}^{2}
\end{gathered}
$$

It should be noted that even in a situation where not all random variables $a_{\theta}(\varpi), C(\varpi), k_{\theta}(\varpi)$ and $f(\varpi)$ (or none of them) are described by the lognormal distribution, the equations (15) and (16) are still true as long as the said components of the random variables are statistically independent from each other. In this situation however it cannot be said that the random variable $N_{\theta}(\varpi)$ is described by the lognormal distribution.

The lognormal distribution is most commonly used to describe the random nature of the material properties of steel, but in the case of small populations it is possible to replace it with a normal distribution. The way to describe characteristic values for distributions that are a combination of independent normal random variables has been specified, among others, by Biegus [2], limiting however detailed solutions to the simplest cases of combination of two independent random variables.

In today's literature, one can find statistics on random variability $C(\varpi)$ and $f(\varpi)$, based on the results of representative surveys, however the Author is not aware of the existence of statistics regarding parameters $a_{\theta}(\varpi)$ and $k_{\theta}(\varpi)$. The ratio of the structural strain/stability model at increased fire temperatures $-a_{\theta}(\varpi)$ is in addition itself a combination of other random parameters, including in particular: random slenderness of the member, imperfection parameter value, accuracy of estimating the temperature of a structural element and the difficulty to determine statistics thereof which reduces the knowledge of characteristics describing other variable components, which greatly complicates the issue.

Thus, the issue of probabilistic assessment of the structural resistance in fire conditions associated, say, with the description of random parameters mentioned above, is becoming a new, interesting and challenging research space for contemporary and future generations of scientists.

\section{Influence of research methods on the values of key strength properties of structural steels}

Knowledge of strength properties of various types of steel at increased temperatures is crucial for a proper understanding of the behaviour of steel and composite structures subjected to fire conditions. Very often, to assess phenomena and evaluate resistance of steel structures to fire simplified material models are used, which in consequence does not always lead to satisfactory and reliable results. In the case of more advanced numerical analysis carried out using e.g. FEA tools, only the adoption of a properly selected material model, based on the actual strength tests, reflecting as precisely as possible the actual processes of degradation of material properties, becomes a guarantee of obtaining reliable results. These strength tests carried out to determine the actual properties of the material must be designed and carried out in compliance with recommendations of codes in relation to the test conditions, in order to be considered as credible and reliable, as well as useful for comparative reasons. A wider review of the constitutive material models of structural steels used in the numerical calculations of structures subjected to fire was made by Kodur et al. in [24]. The authors extensively discussed the impact of discrepancies in the constitutive relations presented on the quality and reliability of the results obtained. Furthermore, they drew attention to the influence of creep strains, which, though usually ignored in structural analyses in normal design conditions, can be an important component of the total deformation of the structure in case of its performance at increased temperature, deciding on its utility values.

\subsection{Preparation of samples, basic methods and types of tests}

\subsubsection{Preparation and dimensions of samples}

Basic principles of static tensile testing of metals at increased temperatures are included in the code [25]. Thickness and width of measurement areas of each sample are dependent on the dimensions (thickness) of the elements they are obtained from, and determined in accordance with the rules adopted in the referenced code.

\subsubsection{Types of material tests at increased temperatures}

There are two well-known methods of testing - the static tensile testing of metals subjected to elevated temperatures: testing in a steady temperature field, commonly known in the literature as "steady-state test" and testing in a transient state, known as "transient-state test".

Steady-state tests consist in the tension of samples previously heated to a predetermined temperature and then subjected to loading. However, this is an unnatural situation, not having much in common with the real conditions of structural performance during fire. In real structures - in the event of a fire, we usually deal with a completely opposite situation - the structure first subject to loads gets exposed to thermal actions of varying rate of change of the temperature field, directly dependent on the dynamics of a fire. Tests that attempt to recreate such cause and effect sequence of events are much more difficult to perform and their results - to interpret. As it turns out, there is an additional parameter that is not without significance for some of the strength properties of steel, namely the heating rate, which depends directly on the dynamics of fire growth, being a value specific for the environment and conditions in the fire compartment, as defined e.g. by the function of the compartment, fuel supply, ventilation conditions, etc. Selected testing of steel sensitivity to the heating rate were conducted among others by Bednarek and Kamocka [26] and Outinen [27]. This article presents only the main findings of these tests. 
Steady-state tests are much easier to carry out in a technical sense and therefore this method is mostly used in many cases, where correct reflecting of the fire scenario is less important and has no significant effect on the analysed values. According to Outinen [27], due to some imperfections in the methods of assessment of strength parameters by steady-state tests, the only fully recognized and recommended approach used in research projects, especially in case of testing low-carbon structural steel, in which the correctness of reflecting phenomena and processes has the highest value, are the tests in the transient thermal conditions (transient-state tests), simulating development of the real fire, though not always taking into account the final phase - the cooling phase, which is normally present in real conditions (extinguishing action, spontaneous extinguishing due to fuel exhaustion or lack of oxygen).

\subsection{Transient-state tests}

In the static tensile test in the transient temperature field, the tested sample remains under a constant load and is subjected to transient in time (but transient at a constant speed increase) action/influence of the temperature field. During the test, temperature and strain are measured directly. Temperature activated elongation is deducted from the total strain measured. The stress-strain curve is created in an indirect way, on the basis of the results transformation, according to the scheme shown in Fig. 4, which can be a source of unintentional mistakes and inaccuracies.

Static tensile test in variable temperature field is performed for specific load levels, corresponding to nominal values of tensile stresses referenced to the original cross section of the tested member: $\sigma_{1}, \sigma_{2}, \sigma_{3}$, (e.g. $\left.80,160,240 \mathrm{~N} / \mathrm{mm}^{2}\right)$. The temperature increase rate in the heated member is adopted according to the standard [25]. Testing continues until the sample ruptures or achieves a specific temperature. For research purposes, it is as a rule assumed that the total member deformation at increased temperatures consists of mechanical deformations resulting from the applied load $-\varepsilon_{e-p}(\sigma, T)$ and thermal deformations $\varepsilon_{T}(T)$. This way of reasoning is a certain simplification of the issue, since in reality, as proposed by the International Committee RILEM-COMMITTEE 44-PHT [25], the total deformation at increased temperatures is described by the Eq. (25):

$$
\varepsilon=\varepsilon_{T}(T)+\varepsilon_{e-p}(\sigma, T)+\varepsilon_{t}(\sigma, T, t)
$$

where:

$\varepsilon_{T}(T)$ - means the thermal elongation $\varepsilon_{T}=\alpha(T) \cdot \Delta T$ resulting from thermal elongation of steel;

$\varepsilon_{e-p}(\sigma, T)$ - is amechanical deformation, calculated excluding creep, and thus formally unrelated to time-dependent effects. This component is generally described by the Ramberg-Osgood equation as follows:

$$
\varepsilon_{e-p}=\varepsilon_{e}+\varepsilon_{p}=\frac{\sigma}{E(T)}+0.002\left[\frac{1}{\sigma_{y}(T)}\right]^{\mu(T)}|\sigma|^{\mu(T)-1} \sigma
$$

$\varepsilon_{t}(\sigma, T, t)$ - is a creep deformation as a function of three variables: stress, temperature and time. A description method depends on the theoretical model adopted.

In practice, in the case of tensile test in anisothermal conditions, the component corresponding to the creep deformation is neglected (inter alia, due to a relatively short time of the member exposure to specific thermal conditions), while deformations resulting from thermal elongation of steel are determined experimentally, by conducting the test with the same parameters of temperature increase, but with a negligible load (e.g. of about $3 \mathrm{~N} / \mathrm{mm}^{2}$ ), or - as a last resort - are determined on the basis of analytical formulas.

Deformation values determined in this way, measured respectively in specific temperature conditions $-T_{1}, T_{2}, T_{3}$ and thus corresponding to a given temperature level of the tested member, are put on the y-axis, whereas corresponding values of stresses that caused them - on the x-axis.

Important mechanical properties of steel, such as the value of the yield stress and Young modulus are determined on the basis of so created stress-strain curve. Tests are performed at different levels of sample stresses and different heating rates. In order to enable comparison of results with each other, they should be referred to tests conducted at the same rate of increase in temperature of the member.

Tests conducted by Bednarek and Kamocka [26] were oriented at the assessment of the impact of differences in the heating rate on the stability of certain values and not at confirming nominal values of important strength parameters given in the structural design standards, hence the tests were made at five different heating speeds between $5^{\circ} \mathrm{C} / \mathrm{min}$ and $65^{\circ} \mathrm{C} / \mathrm{min}$, lower than the generally accepted reference level, which is represented by the curve ISO 834 . Wishing to compare the actual material performance and the description accepted in the standards, a higher rate of the temperature increase should be recreated during testing, reflecting the standard fire conditions and the corresponding temperature increase rate of fire gases and adequate temperature increase rate in the tested member. It is important to measure the temperature of the heated member during the tests of strength parameters of structural materials and not the temperature of fire gases, since these values are different from each other and can lead to subsequent inaccuracies whose source may be hard to detect. In the course of the testing, a clear effect of creep was observed, inversely proportional to the rate of heating (the higher the heating rate, i.e. the higher fire dynamics, the less creep). This confirms the previously mentioned observations made by Kodur, Dwaikat and Fike [24]. 

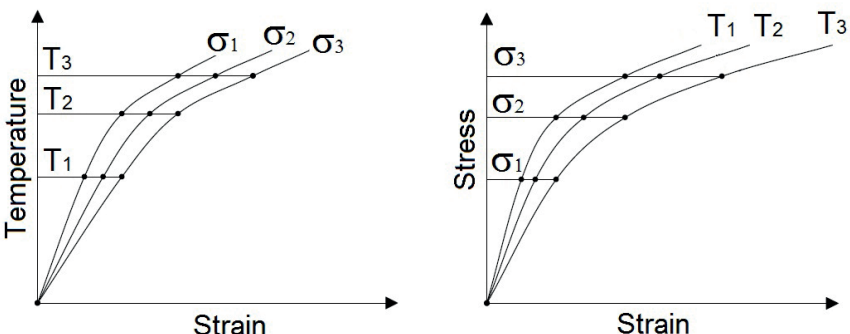

Fig. 4 Converting the stress-strain curves from the transient-state test results

Tests carried out by Bednarek and Kamocka [26] were further oriented to determine the critical temperature $-T_{\text {cr }}$ (the temperature at which a sample is damaged at a given level of load), the temperature for which the total sample relative elongation was $\varepsilon=1 \%-T_{\varepsilon=1 \%}$ and the critical time - $\tau_{\text {cr }}$ (the time measured from the start of the test at $20^{\circ} \mathrm{C}$ until reaching the critical temperature by the sample). Furthermore, measurements were made of the critical strain $-\varepsilon_{\mathrm{cr}}$, which is the sum of the sample strain at the time of rupture in given thermal conditions due to the applied load, according to the sample testing program.

Analysis of the results of tests performed by Bednarek and Kamocka [26] shows that with increasing the heating rate, values of the critical temperature also increase (i.e. the temperature identified with the moment of the structure destruction) and of the total structural strain decreases, thereby increasing (in given temperature conditions) the resistance of the structural member understood as its fire endurance.

Outinen [27] presented, inter alia, results of transient-state tests carried out in order to determine the value of Young modulus. The results of the tests were compared with those that are available in the literature and design standards, as well as, with the results of steady-state tests. A comparative analysis conducted by Outinen showed that the values obtained from experimental tests, regardless of the adopted method, do not differ significantly from the standard curve set out in EN 1993-1-2 [3].

The testing showed that the heating rate of steel also affects the value of the ultimate force measured in the tensile test in transient thermal conditions. With the increase of the heating rate, increase in values of tensile stresses was recorded, corresponding to a given temperature level.

The same results were also obtained some years ago by Anderberg [29]. It is believed that the increased heating rate slows down the thermally activated processes occurring in the internal structure of the material, leading to its damage at high temperatures. The value of the critical temperature increases with the increasing heating rate, while reducing the value of the critical strain, which is also confirmed by the above-cited tests of Bednarek and Kamocka [26]. Sources of such behaviour should be sought in the internal thermal inertia of steel. It is one of its material features.

\subsection{Steady-state tests}

In the case of steady-state tests, the sample tested is heated up to a predetermined temperature level and then subjected to tensile forces, as is the case of testing conducted at room temperature. Both strains and accompanying stresses, in contrast to the transient-state test, are recorded directly. Deformation of the sample during testing is controlled through the load or alternatively - through the displacement. In both cases, increases are applied uniformly. The rate of the strain/load growth is crucial to the results obtained, therefore this parameter must be in full compliance with the regulations of codes in this regard. The higher the tension rate, the higher the resulting value of the ultimate force. Unfortunately, most tests reported in the literature do not contain information on the testing parameters, which makes the results less useful for comparison purposes.

Outinen's [27] tests confirmed correctness of the above observations also in the test conditions at increased temperature, wherein the maximum values of the ultimate force at a given temperature were obtained in the case of steady-state tests conducted at high speed of applied strains, the subsequent ones - for similar thermal conditions and at low speed of applied strains, and the lowest values of the ultimate force - in the case of transient-state tests. In every case, the standardized stress-strain curve, determined in accordance with the rules given in EN 1993-1-2 [3] gave a safe estimation for the assessment of structural safety.

As a result of numerous series of tests performed for different types of steel, it was observed that the impact of variability of the heating conditions and rate of strain application is much greater for carbon steels than in the case of stainless steel.

\section{Influence of material model, geometrical and thermal imperfections on the results of numerical analyses}

Presentation and summary of results of numerical investigation of an axially loaded steel column, subjected to axial and rotational restraints of supports and elevated temperature, simulating fire actions, was included by Kwaśniewski, Król and Łącki in [30]. The paper discusses the outcomes of the parametric study, taking into account uncertainties arising from variability of the steel material model, the size of geometric imperfections and the variation of temperature field along the length of the column. The analyses carried out have shown how seemingly insignificant and difficult to discover model imperfections can lead to misinterpretation of phenomena assessed solely on the basis of numerical calculations, without their proper validation and verification, based on results of a real experiment. 


\section{Summary and final conclusions}

This paper outlines a probabilistic basis for assessing resistance of steel structures during fire. Various sources of uncertainty were pointed out, associated with estimating safety of steel structures and affecting the reliability of the results. Universal factors influencing random structural resistance in all design conditions were separately described, such as, for example, geometrical, technological or material and structural imperfections. A significant part of the paper is devoted to issues that relate only to the accidental situation of fire, with an emphasis on the variability of key material properties of steel as a function of temperature and the rate of its growth. Reference was made to the uncertainty of the model describing the load-carrying resistance of the structure and the Author's own modification of the resistance model applicable for fire temperature conditions was proposed.

The results of the study entitle to formulate a few general broader remarks, related to the issues of structural reliability in the fire situation, and the problems in its resistance assessment by probabilistic methods:

1. Probability of load-carrying resistance loss in the accidental situation of fire should be treated as a random event, resulting directly from the probability of outbreak of developed fire and the probability of exceeding at the same time of a certain limit state, with simultaneous combination of mechanical loads and high temperatures actions, affecting the structure. On the other hand, the probability of outbreak of developed fire is a conditional probability, whose value is the sum of the probability of starting a fire and the product of the probability of a lack of effectiveness of fire-protection measures (understood as consistent operation of automatic fire-detection and fire-fighting systems and emergency services) and the likelihood of failure to suppress the flames before the flashover. Having only the knowledge of statistics relating closely to the material parameters and load-carrying resistance, it is in fact impossible to properly assess the risk of structural failure and the level of structural safety with the use of probabilistic methods, without the knowledge of statistics on fire starting relevant to the function/type of the building to which they relate and statistics on effectiveness of the services or systems. The problem is therefore a complex mathematical task, requiring not only a thorough knowledge of probability theory but also an access to relevant statistical data, which, absolutely necessary to carry out fully credible analyses, are currently hardly available and incomplete.

2. The self-phenomenon of fire, within its physical meaning is very complex itself whose course and effects on the structures are highly dependent on environmental conditions. Fire is a phenomenon difficult to describe in terms of analysis and the available models are inconsistent and can lead to incorrect estimates. In every case, it causes a change in environmental conditions, resulting in a non-linear behaviour of the structure. Nonlinearity of phenomena highly complicates the analyses and intensifies the effect of different types of imperfections that affect the actual load-carrying capacity of the structure. Moreover, the way of fire growth (its dynamics) itself significantly affects the magnitude of strength parameters of material. With increasing temperature, both steel yield strength value and elastic modulus value are reduced, being the key values in the adopted code procedures of structural safety assessment - both in normal design conditions and in the accidental design situation of fire.

3. To conduct a probabilistic assessment of structural resistance under fire, it is necessary to know the statistics on the aforementioned utility parameters (characteristics), describing the fire environment (such as e.g. fire load density corresponding to a given function of premises, the size of the fire compartment, the ratio of openings, thermal parameters of construction materials and separating enclosures, etc.), giving information on the magnitude of the mean value, variance and distribution type. In addition, it is necessary to know the corresponding data relating to the temperaturedependent parameters used in the available, code-based assessment procedures of structures, such as the random reduction factor of random structural strength $-k_{\theta}(\varpi)$ or the random coefficient of the structural strain/stability model at the temperature of fire $-a_{\theta}(\varpi)$. At the moment, some statistics on the fire performance parameters characteristics can be found mainly in American and to a lesser extent - in the Antipodean or Far East reports of surveys. A part of the data is, however, inaccurate due to the specificity of solutions used on these markets and has limited usefulness in European conditions. European statistics are extremely poor and many countries do not collect them at all.

4. A unique part of the work includes a method of describing the model of the random structural resistance in case of fire, represented by the Eqs. (3), (4), (10), and (11), being an innovative and original Author's modification of models used today to describe the random structural resistance under normal conditions. Due to the unique nature of this proposal, for obvious reasons there are also no statistics that would allow the assessment of the variability of $T(\varpi), D(\varpi)$ and the aforementioned $-a_{\theta}(\varpi)$ or $k_{\theta}(\varpi)$, enabling a probabilistic assessment of the structural resistance in fire conditions. Based on the Author's investigation and analysis, there is no substantial or popular evidence demonstrating an individual or body has ever conducted appropriate surveys that could at least to some extent contribute to the formation of such statements. From a research aspect, this issue opens up a whole new chapter in this field.

5. As the analysis of the experimental testing results shows, steel is a structural material sensitive to heating rate and the speed of loading or deformation. It was found, among 
others, that the deformation of the structure due to creep is inversely proportional to the rate of heating. Since the construction and technical regulations generally specify correspondingly higher fire resistance requirements in relation to the main load-bearing structural members, in the practical design, this usually translates into a better (more efficient) protection of this category of elements from the influence of fire. That results in slower heating, which can lead to greater deformation of these members, caused by creep. This justifies the need to pay special attention to this problem at the stage of designing these members.

6. The example of numerical simulations cited in chapter 7 shows how imprecise input data and pre-made simplifying assumptions can affect the quality of the results. In the case of analyses concerning complex and highly nonlinear phenomena, one must have proper technical expertise and theoretical knowledge enabling correct evaluation of the results. Especially in the case of fire analyses, computing systems available on the market offer extremely high potential and provide an excellent tool for research and evaluation of phenomena, however only in a qualitative sense. In terms of quantity, however, their credibility at this stage can be considered as moderately limited. This is because calibration of an actual structure model based on limited results from laboratory tests carried out with the use of selected structural members, does not have to lead to reliable estimates referred to the entire superstructure, consisting of a system of single elements connected to each other in a way that limits the freedom of displacements and deformations.

\section{Acknowledgement}

This article was written during the author's stay at Auburn University, Alabama, USA within the framework of a visiting research scholarship, funded by the Polish-American Fulbright Commission.

The author would like to express his gratitude to the Authorities and the Scientific Council of the Commission for all the support, allowing the integration of Polish and American academics, which globally contributes to improving and strengthening Poland's position in the world and a better understanding of mutual problems and needs.

\section{References}

[1] European Committee for Standardization. "EN 1993-1-1:2005. Eurocode 3: Design of steel structures, Part 1-1: General rules and rules for buildings.” CEN, Brussels. 2005. URL: https://law.resource.org/pub/ eu/eurocode/en.1993.1.1.2005.pdf

[2] Biegus, A. "Probabilistyczna analiza konstrukcji stalowych." (Probabilistic analysis of steel structures.) 197 p. Wydawnictwo Naukowe PWN, Warszawa. 1999. (in Polish) URL: http://www.kkm.pwr. wroc.pl/BIBLIOTEKA/Biegus\%20A.\%20-\%20Probabilistyczna\%20 analiza.pdf
[3] European Committee for Standardization. "EN 1993-1-2:2005. Eurocode 3: Design of steel structures, Part 1-2: General rules. Structural fire design.” CEN, Brussels, 2005. URL: https://law.resource.org/pub/eu/ eurocode/en.1993.1.2.2005.pdf

[4] American Institute of Steel Construction. "Specification for Structural Steel Buildings.” ANSI/AISC 360-10. An American National Standard, Chicago, June 22, 2010. URL: https://docs.google.com/file/d/0B-pXeGd iQqo2ZmI1YjM4NmQtYjVkNy00MGMxLTgzZmEtODJmZDEzZjMz MGI0/edit

[5] American Institute of Steel Construction. "AISC Steel Design Guide 19. Fire Resistance of Structural Steel Framing.” Chicago, 2003.

[6] New Zealand Standard. "Steel Structures Standard. Part 1: Materials, fabrication, and construction.” NZS 3404, Wellington. 1997. URL: https:// shop.standards.govt.nz/catalog/3404.1:2009(NZS)LOOSELEAF/scope

[7] Polski Komitet Normalizacyjny. „Konstrukcje stalowe. Obliczenia statyczne i projektowanie.” (Steel structures. Design Rules.) PN-B03200:1990, PKN - Polish Committee of Standardization, Warszawa. 1990. (in Polish) URL: http://sklep.pkn.pl/pn-b-03200-1990p.html

[8] European Convention for Constructional Steelwork. "Design manual on the European recommendations for the fire safety of steel structures." ECCS-TC3:1985. Technical Note No.35, ECCS, Brussels. 1985.

[9] Lee, J. H., Mahendran, M., Mäkeläinen, P. "Prediction of mechanical properties of light gauge steels at elevated temperatures." Journal of Constructional Steel Research. 59 (12), pp. 1517-1532. 2003. DOI: 10.1016/S0143-974X(03)00087-7

[10] Caro, T. C., Milke, J. A. "A survey of fuel loads in contemporary office buildings." National Institute of Standards and Technology, Report No. NIST-GCR-96-697, Gaithersburg, MD. 1995. URL: http://fire.nist.gov/ bfrlpubs/fire96/PDF/f96080.pdf

[11] Culver, C. G. "Survey results for fire loads and live loads in office buildings.” National Bureau of Standards, Washington, D.C. 1976.

[12] Yii, H. W. "Effect of Surface Areas and Thickness on Fire Loads." Fire Engineering Research Report 200/13, University of Canterbury, Christchurch, New Zealand. 2000.

[13] Hasofer, A. M., Lind, N. "An exact and invariant first-order reliability format.” Journal of Engineering Mechanics. 100, pp. 111-121. 1974.

[14] Rackwitz, R., Fiessler, B. "Structural reliability under combined random load sequences." Computers and Structures. 9 (5), pp. 489-494. 1978. DOI: 10.1016/0045-7949(78)90046-9

[15] Thoft-Christensen, P., Baker, M.J. "Structural reliability theory and its applications." 267 p. Springer-Verlag, New York. 1982. DOI: 10.1007/978-3-642-68697-9

[16] Thoft-Christensen, P., Murotsu, Y. "Application of structural systems reliability theory." 343 p. Springer-Verlag, New York. 1986. DOI: 10.1007/978/3-642-82764-8

[17] Nowak, A. S., Collins, K. R. "Reliability of Structures". $2^{\text {nd }}$ Edition, 391 p. CRC Press, Taylor \& Francis Group, New York. 2013.

[18] Melchers, R. E. "Structural reliability analysis and prediction." $437 \mathrm{p}$ $2^{\text {nd }}$ Edition, John Wiley \& Sons, New York. 2002.

[19] Maślak, M. „Trwałość pożarowa stalowych konstrukcji prętowych.” (Fire resistance of steel bar structures). 203 p. Monograph 370, Series "Civil Engineering", Publishing House of Cracow University of Technology, Cracow. 2008. (in Polish) URL: http://suw.biblos.pk.edu.pl/ resourceDetails\&rId=3984

[20] Ellingwood, B., Galambos, T. V., MacGregor, J. G., Cornell, C. A. "Development of a probability based load criterion for American National Standard A58." National Bureau of Standrads, Washington, DC. NBS Special Publication 577. 1980. URL: http://nvlpubs.nist.gov/ nistpubs/Legacy/SP/nbsspecialpublication577.pdf 
[21] Ellingwood, B., MacGregor, J. G., Galambos, T. V., Cornell, C. A. "Probability based load criteria: Load factors and load combinations." Journal of the Structural Division. 108 (5), pp. 978-997. 1982. URL: http://cedb.asce.org/CEDBsearch/record.jsp?dockey $=0034202$

[22] Galambos, T. V., Ellingwood, B., MacGregor, J. G., Cornell, C. A. "Probability based load criteria: Assessment of current design practice." Journal of the Structural Division. 108 (5), pp. 959-977. 1982. URL: http://cedb.asce.org/CEDBsearch/record.jsp?dockey=0034201

[23] Woliński, Sz., Wróbel, K. „Niezawodność konstrukcji budowlanych.” (Reliability of Building Structures.) 184 p. Publishing House of Rzeszów University of Technology, Rzeszów. 2001. (in Polish)

[24] Kodur, V., Dwaikat, M., Fike, R. "High-temperature properties of steel for fire resistance modeling of structures." Journal of Materials in Civil Engineering. 22 (5), pp. 423-434. 2010. DOI: 10.1061/(ASCE)MT.19435533.0000041

[25] International Organization for Standardization. "EN ISO 6892-2:2011. Metallic materials. Tensile testing. Part. 2: Method of test at elevated temperature." ISO, 2011. URL: http://www.iso.org/iso/catalogue_detail. htm? csnumber $=42610$
[26] Bednarek, Z., Kamocka, R. "The heating rate impact on parameters characteristic of steel behaviour under fire conditions." Journal of Civil Engineering and Management. XII (4), pp. 269-275. 2006. DOI: 10.1080/13923730.2006.9636403

[27] Outinen, J. "Mechanical properties of structural steels at high temperatures and after cooling down." Laboratory of Steel Structures Publications 32, Espoo: Helsinki University of Technology. 2007.

[28] Anderberg Y. "Behaviour of Steel at High Temperatures." RILEM 44-PHT, Division of Building Fire Safety and Technology, Report LUTVDG/TVBB-3008, Lund, Sweden. 1983.

[29] Anderberg, Y. "Modelling Steel Behaviour." Fire Safety Journal. 13 (1), pp. 17-26. 1988. DOI: 10.1016/0379-7112(88)90029-X

[30] Kwaśniewski, L., Król, P., Łącki. K. "Virtual tests on axially and rotationally restrained steel column under fire." Journal of Structural Fire Engineering. 2(2), pp. 109-121.2011. DOI:10.1260/2040-2317.2.2.109 